\title{
A STABILITY ANALYSIS OF TYPHOID DISEASE MODEL WITH TREATMENT AND BACTERIA COMPARTMENTS
}

\section{NAGA SOUNDARYA LAKSHMI V.S.V ${ }^{1}$ AND A SABARMATHI ${ }^{*}$}

1: Research Scholar, Department of Mathematics, Auxilium College, Vellore - 632006, Tamilnadu, India

2: Assistant Professor, Department of Mathematics, Auxilium College, Vellore - 632006,

Tamilnadu, India

*Corresponding Author: Naga soundarya lakshmi V.S.V: E Mail: san9sak14@gmail.com

Received 28 ${ }^{\text {th }}$ March 2021; Revised 30 ${ }^{\text {th }}$ April 2021; Accepted $15^{\text {th }}$ June 2021; Available online $1^{\text {st }}$ Oct. 2021

\section{https://doi.org/10.31032/IJBPAS/2021/10.10.5680}

\begin{abstract}
A SIR model with treatment class and bacteria class for Typhoid was formulated. Existence and uniqueness of the solution of the model is verified. The basic reproduction number is calculated. The local stability of disease free equilibrium and endemic equilibrium were verified. Numerical analysis was carried out for the data of Odisha. The graphs were plotted to visualize the stability behaviour of the state variables for different values of parameters.

Keywords: SIR model, Typhoid, stability analysis, basic reproduction number. \section{INTRODUCTION}

Typhoid fever is a very common severe in the second week. The duration of the disease in India which is at its peak in illness is from 4 to 6 weeks. In India, monsoon season. It is caused by the ingestion of contaminated food and water containing Bacteria Salmonella typhi. The main symptoms of Typhoid are high fever, head ache, weakness and diarrhea.

Usually, the symptoms develop after vaccination for Typhoid is available in three forms namely, injection, oral vaccine and conjugate vaccine.

A classical SIR model with a bacteria compartment is given by SIRB model. SIRB model is used to represent the disease spreads
\end{abstract} one or two weeks of exposure, it becomes 
through a bacterium such as Cholera and Typhoid.

Mathematicians used this model for analyzing of such diseases. Olcay Akman [8] formulated different models with bacteria class for Cholera. Nthiiri [7] formulated and analyzed the stability of Typhoid by PSIT model. Getachew [4] studied the stability of SIRB model with carrier state for Typhoid. Peter [9] studied the dynamics of Typhoid fever with SITR model with protected class. Hamadjam Abboubakar [6] derived a SECIR model with vaccination and bacteria compartment for Typhoid and also analyzed the model with control strategies. Fitriyani [3] analyzed the stability of SIRB model for Cholera with vaccination and disinfection.

In this paper, we formulated SIR model with treatment class and bacteria class for Typhoid, including the infectious rate from humans and the infectious rate from environment. For the numerical analysis, we used the data of Odisha, India.

\section{METHODOLOGY}

\section{Formulation of Model}

The following Figure 1 shows the transistion of Typhoid disease with treatment and bacteria compartment.

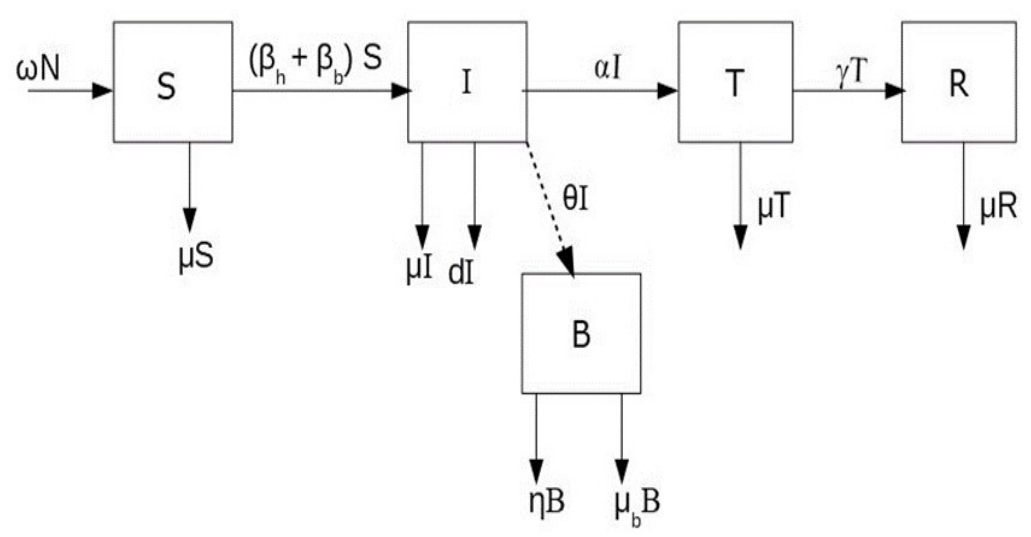

Figure 1: SITRB model for Typhoid disease

The following system of ordinary differential equation represents the Typhoid model,

$$
\begin{gathered}
\frac{d S}{d t}=\omega N(t)-\left(\beta_{h}+\beta_{b}\right) S(t)-\mu S(t) \\
\frac{d I}{d t}=\left(\beta_{h}+\beta_{b}\right) S(t)-(\alpha+\mu+d) I(t) \\
\frac{d T}{d t}=\alpha I(t)-(\gamma+\mu) T(t) \\
\frac{d R}{d t}=\gamma T(t)-\mu R(t) \\
\frac{d B}{d t}=\theta I(t)-\left(\eta+\mu_{b}\right) B(t)
\end{gathered}
$$


With the initial conditions as $\mathrm{S}(\mathrm{t}), \mathrm{I}(\mathrm{t}), \mathrm{T}(\mathrm{t}), \mathrm{R}(\mathrm{t}), \mathrm{B}(\mathrm{t}) \geq 0$, and $\omega_{v} \mu, \mu_{b^{3}} d_{n} \beta_{h^{0}} \beta_{b}, \alpha_{n}, \theta, \eta, \gamma>0$.

Where $S(t), I(t), T(t), R(t), B(t)$ are the Susceptible state, Infectious state, Treatment state, Recovery state and Bacteria state respectively, $\omega$ - Average birth rate, $\mu$ - Average death rate, $\mu_{b}$ - Death rate of bacteria, $\beta_{h}$ - Transistion infection rate from humans, $\beta_{b}$ - Transistion infection rate of bacteria from environment, $\alpha$ - Treatment rate, $\theta$ - Rate of contribution from an infected human, $\eta$ - Disinfection rate, $\gamma$ - Recovery rate, $\mathbf{d}$ - disease induced death rate, $\mathbf{N}$ - Total Population.

\section{Existence and Uniqueness of Solution}

To prove the existence and uniqueness of the solution of the system (1) we use the following theorem and lemma.

\section{Theorem 3.1. Existence and Uniqueness Theorem}

Let $\frac{d y}{d x}=f(x, y), y\left(x_{0}\right)=y_{0} \quad$ and $\quad \mathrm{f}(\mathrm{x}, \quad \mathrm{y})$ be continuous on a domain $\left\{\mathrm{D}=(\mathrm{x}, \mathrm{y}) ; x_{0}-a<x_{0} a ; y_{0}-b<y<y_{0}+b\right\}$ with the Lipschitz condition $\left\|f\left(x, y_{1}\right)-f\left(x, y_{2}\right)\right\| \leq K\left\|y_{1}-y_{2}\right\|$,where $\mathrm{K}$ is a positive integer, then there exist bounded solution in D.

Furthermore, if $\mathrm{f}(\mathrm{x}, \mathrm{y})$ is Lipschitz continuous with respect to $\mathrm{y}$ on a rectangle $R=\left\{(x, y) ; x_{0}-c<x<x_{0}+G ; y_{0}-b<y<y_{0}+b ; c<a\right\}$ then there is a unique solution $y(t)$ in $R$.

Lemma 3.2. If $\mathrm{f}(\mathrm{x}, \mathrm{y})$ has continuous partial derivative $\frac{\partial f_{i}}{\partial y_{j}}$ on a bounded closed domain $\mathrm{R}$, is a set of real numbers. Then it satisfies a Lipschitz condition in R.

\section{RESULTS AND DISCUSSIONS}

Proof: To prove the main theorem, from (1) we consider the following:

$$
\begin{aligned}
& f_{1}=\omega N(t)-\left(\beta_{h}+\beta_{b}\right) S(t)-\mu S(t) \\
& f_{2}=\left(\beta_{h}+\beta_{b}\right) S(t)-(\alpha+\mu+d) I(t) \\
& f_{3}=\alpha I(t)-(y+\mu) T(t) \\
& f_{4}=\gamma T(t)-\mu R(t) \\
& f_{5}=\theta I(t)-\left(\eta+\mu_{b}\right) B(t)
\end{aligned}
$$

Now we show that the partial derivatives, $\frac{\partial f_{i}}{\partial y_{j}}, i, j=1,2,3,4,5$ are bounded and continuous. We derive the following partial derivatives for the model equations (2) - (6). We have

$$
\begin{aligned}
& \frac{\partial f_{1}}{\partial S}, \frac{\partial f_{1}}{\partial I}, \frac{\partial f_{1}}{\partial T}, \frac{\partial f_{1}}{\partial R}, \frac{\partial f_{1}}{\partial B}<\infty \\
& \frac{\partial f_{2}}{\partial S}, \frac{\partial f_{2}}{\partial I}, \frac{\partial f_{2}}{\partial T}, \frac{\partial f_{2}}{\partial R}, \frac{\partial f_{2}}{\partial B^{*}}<\infty \\
& \frac{\partial f_{3}}{\partial S}, \frac{\partial f_{3}}{\partial I}, \frac{\partial f_{3}}{\partial T}, \frac{\partial f_{3}}{\partial R}, \frac{\partial f_{3}}{\partial B^{*}}<\infty
\end{aligned}
$$




$$
\begin{aligned}
& \frac{\partial f_{4}}{\partial S}, \frac{\partial f_{4}}{\partial I}, \frac{\partial f_{4}}{\partial T}, \frac{\partial f_{4}}{\partial R}, \frac{\partial f_{4}}{\partial B}<\infty \\
& \frac{\partial f_{5}}{\partial S}, \frac{\partial f_{5}}{\partial I}, \frac{\partial f_{5}}{\partial T}, \frac{\partial f_{5}}{\partial R}, \frac{\partial f_{5}}{\partial B}<\infty
\end{aligned}
$$

It is clear that the above partial derivatives are continuous and bounded. Hence, there exists a unique solution of (1) in the region D which completes the proof.

\section{Equilibrium Analysis}

Case (1): Disease free equilibrium

Let $\bar{S}$ be the positive solution of $\frac{d S}{d t}=0$

From (1), $\bar{s}=\frac{\omega N}{\mu+\beta_{h}+\beta_{b}}$

Case (2): Endemic Equilibrium

Let $S^{*}{ }_{n} I^{*}, T^{*}, R^{*}, B^{*}$ be the positive solutions of

$$
\frac{d S}{d t}=0, \frac{d I}{d t}=0, \frac{d T}{d t}=0, \frac{d R}{d t}=0, \frac{d B}{d t}=0
$$

From the model equation (1),

$$
\begin{gathered}
S^{*}=\frac{\omega N}{\mu+\beta_{h}+\beta_{b}} \\
I^{*}=\frac{\beta_{h}+\beta_{b}}{\alpha+\mu+d} \frac{\omega N}{\mu+\beta_{h}+\beta_{b}} \\
T^{*}=\frac{\alpha}{\gamma+\mu} \frac{\beta_{h}+\beta_{b}}{\alpha+\mu+d} \frac{\omega N}{\mu+\beta_{h}+\beta_{b}} \\
R^{*}=\frac{\gamma}{\mu} \frac{\alpha}{\gamma+\mu} \frac{\beta_{h}+\beta_{b}}{\alpha+\mu+d} \frac{\omega N}{\mu+\beta_{h}+\beta_{b}} \\
B^{*}=\frac{\theta}{\eta+\mu_{b}} \frac{\beta_{h}+\beta_{b}}{\alpha+\mu+d} \frac{\omega N}{\mu+\beta_{h}+\beta_{b}}
\end{gathered}
$$

Hence, the endemic equilibrium is

$$
\begin{gathered}
\left(S^{*}{ }_{s}^{*}{ }_{n}^{*} T^{*}{ }_{s} R^{*}, B^{*}\right)=\left(\frac{\omega N}{\mu+\beta_{h}+\beta_{b}}, \frac{\beta_{h}+\beta_{b}}{\alpha+\mu+d} \frac{\omega N}{\mu+\beta_{h}+\beta_{b}},\right. \\
\frac{\alpha}{\gamma+\mu} \frac{\beta_{h}+\beta_{b}}{\alpha+\mu+d} \frac{\omega N}{\mu+\beta_{h}+\beta_{b}}, \frac{\gamma}{\mu} \frac{\alpha}{\gamma+\mu} \frac{\beta_{h}+\beta_{b}}{\alpha+\mu+d} \frac{\omega N}{\mu+\beta_{h}+\beta_{b}}, \\
\left.\frac{\theta}{\eta+\mu_{b}} \frac{\beta_{h}+\beta_{b}}{\alpha+\mu+d} \frac{\omega N}{\mu+\beta_{h}+\beta_{b}}\right)
\end{gathered}
$$

\section{Basic Reproduction Number}

We know the largest Eigen value of next generation matrix $\mathrm{FV}^{-1}$ is the basic reproduction number [1].

From (1) we have, 


$$
\begin{gathered}
F=\left[\begin{array}{cc}
\left(\beta_{k}+\beta_{b}\right) S & 0 \\
\theta & 0
\end{array}\right], \quad \boldsymbol{V}=\left[\begin{array}{cc}
\alpha+\mu+d & 0 \\
0 & \eta+\mu_{b}
\end{array}\right] \\
F V^{-1}=\left[\begin{array}{cc}
\frac{\left(\beta_{h}+\beta_{b}\right) S}{\alpha+\mu+d} & 0 \\
\frac{\theta}{\alpha+\mu+d} & 0
\end{array}\right]
\end{gathered}
$$

Hence, we have

$$
R_{0}=\frac{\beta_{h}+\beta_{b}}{\alpha+\mu+d}
$$

\section{Stability Analysis}

Theorem 6.1. The disease free equilibrium is locally asymptotically stable if $R_{0}<1,[9]$

Proof::

The Jacobian matrix of (1) is

$$
I=\left[\begin{array}{ccccc}
-\left(\mu+\beta_{h}+\beta_{b}\right) & 0 & 0 & 0 & 0 \\
\beta_{h}+\beta_{b} & -(\alpha+\mu+d) & 0 & 0 & 0 \\
0 & \alpha & -(\gamma+\mu) & 0 & 0 \\
0 & 0 & \gamma & -\mu & 0 \\
0 & \theta & 0 & 0 & -\left(\eta+\mu_{b}\right)
\end{array}\right]
$$

The characteristic equation of $(8)$ is

$$
\left[-\left(\mu+\beta_{h}+\beta_{b}\right)-\lambda\right][-(\alpha+\mu+d)-\lambda][-(\gamma+\mu)-\lambda][-\mu-\lambda]\left[-\left(\eta+\mu_{b}\right)-\lambda\right]
$$

The Eigen values of (9) are

$\lambda_{1}=-\left(\mu+\beta_{k}+\beta_{b}\right)<0$

$\lambda_{2}=-(\alpha+\mu+d)<0$

$\lambda_{3}=-(y+\mu)<0$

$\lambda_{4}=-\mu<0$

$\lambda_{5}=-\left(\eta+\mu_{b}\right)<0$

From (7), R0<1.

Hence the disease free equilibrium is locally asymptotically stable.

Theorem 6.2. The endemic equilibrium is locally asymptotically stable if R0 $>1$ [7] Proof. We prove this theorem, using trace and determinant of the Jacobian matrix $J$. The trace of $(8)$ is

$$
\begin{gathered}
\operatorname{tr}(J)=\left[-\left(\mu+\beta_{h}+\beta_{b}\right)\right]+[-(\alpha+\mu+d)]+[-(\gamma+\mu)]+[-\mu]+\left[-\left(\eta+\mu_{b}\right)\right] \\
=-\gamma-4 \mu-\eta-\mu_{b}-\alpha-d-(\alpha+\mu+d) R_{0}
\end{gathered}
$$

Hence the trace of $\mathrm{J}$ is negative, when $R_{0}>1$

The determinant of (8) is

$|J|=\mu(\alpha+\mu+d)(\gamma+\mu)\left(\eta+\mu_{b}\right)\left[-\left(\mu+\beta_{n}+\beta_{b}\right)\right]$

Here $|J|>0$, with the condition $\mu(\alpha+\mu+d)(\gamma+\mu)\left(\eta+\mu_{b}\right)>0$.

Hence the trace of $\mathrm{J}$ is negative and determinant of $\mathrm{J}$ is positive. Thus the endemic equilibrium is locally asymptotically stable if R0 $>1$

\section{Numerical Analysis}

For Odisha, $\omega=0.0526 ; \mu=0.0208 ; \mathrm{d}=0.00047$, From [5], $\mu_{b}=0: 001$ and let us assume

$\beta_{h}=0: 019 ; \beta_{b}=0: 019 ; \alpha=0: 02 ; \theta=0: 009 ; \eta=0: 02 ; \gamma=0: 4$

From (7), R0 $=0: 9208$, which is stable in disease free equilibrium as $\mathrm{R}_{0}<1$. Hence the basic reproduction value for Typhoid in Odisha is 0.9208 . 
Figure (2) shows the flow of susceptible, Infectious, Treatment, Recovered and Bacteria state variables with respect to time. Figure (3) and Figure (4) shows the flow of susceptible class for different values of $\beta_{h}$ and $\beta_{b}$ respectively. And the individual of susceptible class decreases whenever infectious rates $\beta_{h}$, $\beta_{b}$ increases.

Figure (5) shows the flow of infectious class for different values of $\alpha$ and the individual of infectious class decreases whenever the treatment rate increases.
Figure (6) shows the flow of treatment class for different values of $\gamma$ and the individuals of treatment class increases whenever the recovery rate decreases. Figure (7) shows the flow of Recovered class for different values of $\gamma$ and the individuals of recovered class increases whenever the recovery rate increases. Figure (8) shows the flow of Bacteria class for different values of $\eta$ and the individuals of bacteria class increases whenever the disinfection rate increases.

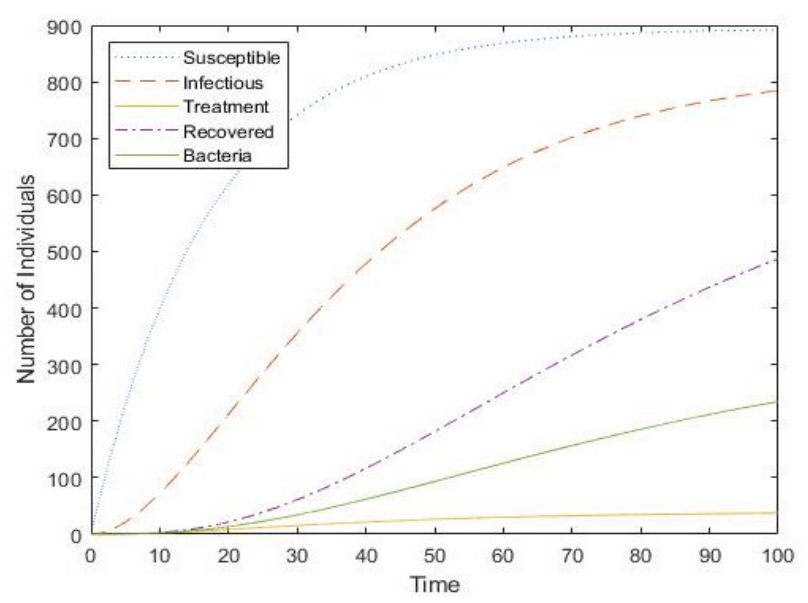

Figure 2: Transmission of Typhoid in Odisha

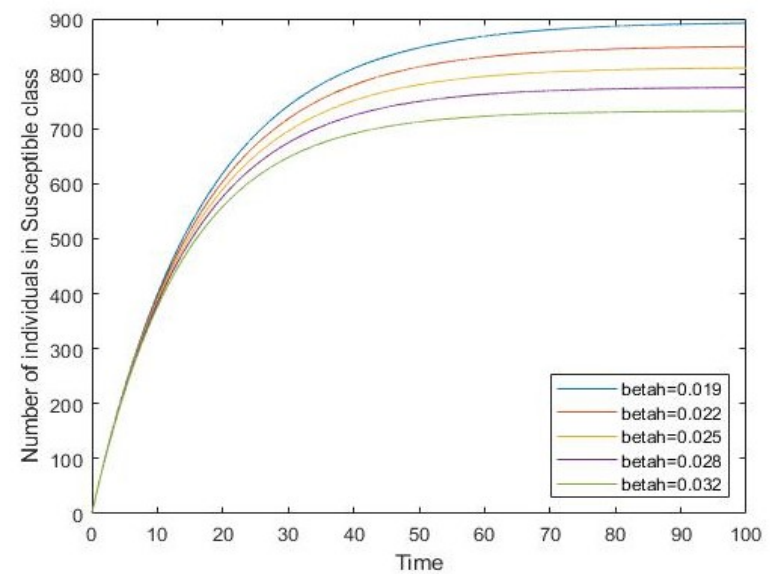

Figure 3: Flow of Susceptible class for different values of $\boldsymbol{\beta}_{h}$ 


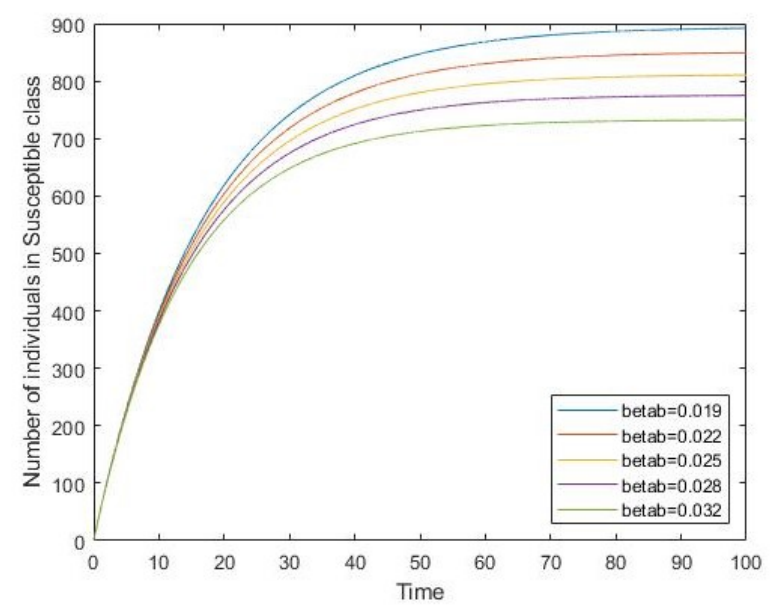

Figure 4: Flow of Susceptible class for different values of $\beta_{b}$

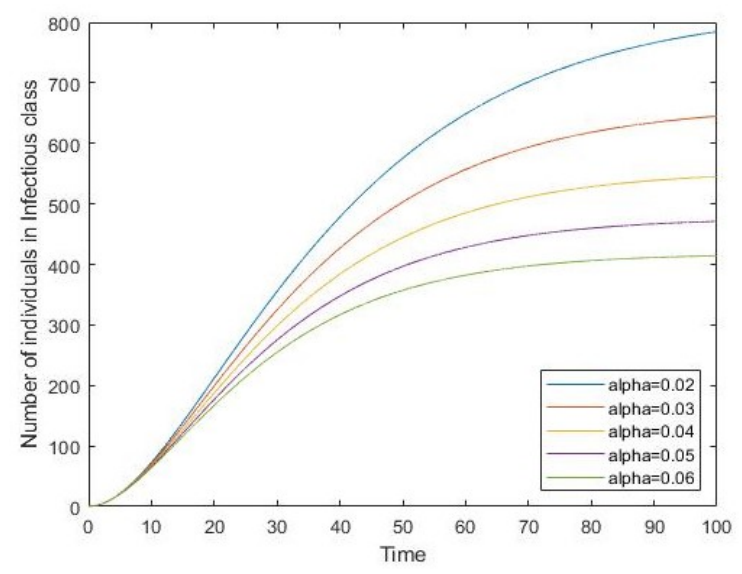

Figure 5: Flow of Infectious class for different values of $\alpha$

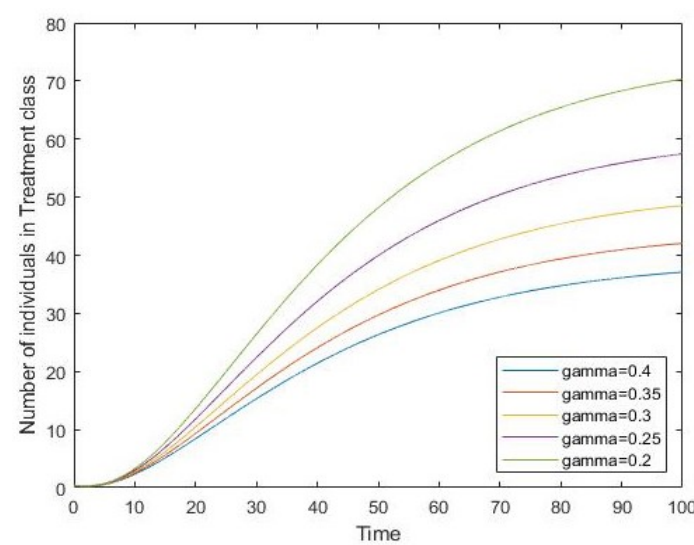

Figure 6: Flow of Treatment class for different values of $\gamma$ 


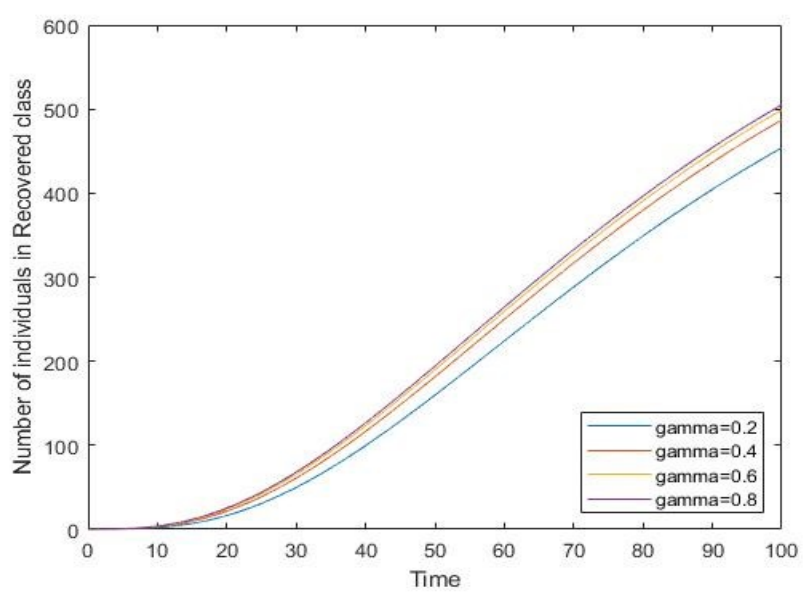

Figure 7: Flow of Recovered class for different values of $\gamma$

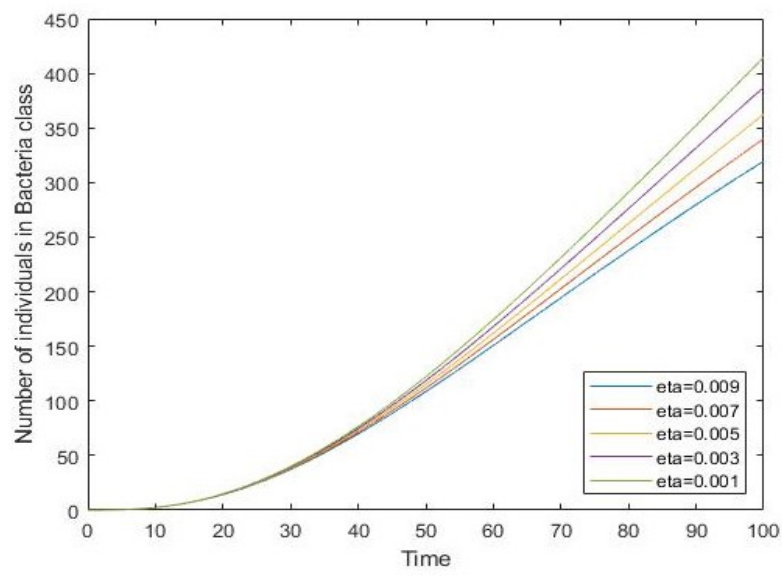

Figure 8: Flow of Bacteria class for different values of $\boldsymbol{\eta}$

\section{CONCLUSION}

A Typhoid model with treatment class and bacteria class was formulated. Existence ad uniqueness of the solution of the model is verified. The basic reproduction number $\mathrm{R}_{0}$ is calculated. Using $\mathrm{R}_{0}$, the local stability of disease free equilibrium when $\mathrm{R}_{0}$ $<1$ and endemic equilibrium when $\mathrm{R}_{0}>1$ were verified. For Odisha in India, the $\mathrm{R}_{0}$ is calculated as 0.9208 , which is locally stable in disease free equilibrium. The graphical study on state variables was demonstrated.
Particularly, it is observed that the individuals in treatment class decreases whenever the treatment rate increases and the individuals in bacteria class increases whenever the disinfection rate decreases.

Conflict of Interest: Nil Source of Funding: This study has not received any funding from any funding agency

\section{REFERENCES}

[1] O. Diekmann, J. A. P. Heesterbeek, and M. G. Roberts (2010). The 
construction of next-generation

matrices for compartmental

epidemic models, J. R. Soc.

Interface 7, 873-885.

[2] Enteric Fever (Typhoid) -

Prevalence and Deaths in India,

Medindia,

https://www.medindia.net/health_st

atistics/diseases/typhoid-enteric-

fever-india-healthstatistics.asp

(Accessed on 20 August, 2021).

[3] A. Fitriyai (2020). Stability analysis of mathematical model (sirb) in the spread of cholera with vaccination and disinfection, Journal of Physics: Conference Series 1524, 012053.

[4] Getachew Teshome Tilahun, Oluwole Daniel Makinde, and David Malonza (2017). Modelling and Optimal Control of Typhoid Fever Disease with CostEffective Strategies, Computational and Mathematical Methods in Medicine, Volume 2017, Article ID 2324518, https://doi.org/10.1155/2017/2324518

[5] M. Gosh, P. Chandra, P. Sinha, and J. B. Shukla (2006). Modelling the spread of bacterial infectious disease with environmental effect in a logistically growing human population, Non-linear Analysis: Real World Applications, 7(3), 341-363.

[6] Hamadjam Abboubakar and Reinhard Racke (2019). Mathematical modelling and optimal control of typhoid fever.
Konstanzer Schriften in Mathematik, $\mathrm{Nr}$. 386: 1-30.

(https://kops.uni-

konstanz.de/bitstream/handle/123456789/477 68/Abboubakar_2-

ise8u21e4ib13.pdf? sequence $=3$ \&isAllowed $=$ y)

[7] J. K. Nthiiri, G. O. Lawi, C. O. Akinyi, D. O. Oganga, W. C. Muriuki, M. J. Musyoka, P. O. Otieno and L. Koech (2016). Mathematical Modelling of Typhoid Fever Disease Incorporating Protection against Infection, British Journal of Mathematics and Computer Science, 14(1), 1-10.

[8] Olcay Akman, Marina Romadan Corby and Elsa Schaefer (2016). Examination of models for cholera: insights into model comparison methods, Letters in Biomathematics, 3(1), 93-118.

[9] Peter, O.J, Ibrahim, M.O., Akinduko, O.B, Rabiu, M (2017). Mathematical Model for the Control of Typhoid Fever, IOSR Journal of Mathematics, 13(4), 6066. 